\title{
Efficient Robust Adaptive Beamforming for Cyclostationary Signals
}

\author{
Ju-Hong Lee, Yung-Ting Lee, and Wen-Hao Shih
}

\begin{abstract}
This paper deals with the problem of robust adaptive array beamforming for cyclostationary signals. By exploiting the signal cyclostationarity, the LS-SCORE algorithm presented in a paper by Agee et al.has been shown to be effective in performing adaptive beamforming without requiring the direction vector of the desired signal. However, this algorithm suffers from severe performance degradation even if there is a small mismatch in the cycle frequency of the desired signal. In this paper, we first evaluate the performance of the LS-SCORE algorithm in the presence of cycle frequency error (CFE). An analytical formula is derived to show the behavior of the performance degradation due to CFE. An efficient method is then developed based on the fact that the array output power approaches its maximum as the CFE is reduced. We formulate the problem as an optimization problem for reducing the CFE iteratively to achieve robust adaptive beamforming against the CFE. Simulation examples for confirming the theoretical analysis and showing the effectiveness of the proposed method are provided.
\end{abstract}

Index Terms-Adaptive beamforming, cycle frequency error, cyclostationary signals.

\section{INTRODUCTION}

$\mathbf{F}$ OR A STEERED-beam adaptive beamformer, the adaptive weights are calculated by minimizing the beamformer's output power subject to the constraint that forces the array to make a constant response at the steering direction [1]. Hence, the performance of the beamformer is very sensitive to the accuracy of the steering vector. It has been shown that the mismatch between the direction vector of the desired signal and the steering vector causes severe performance degradation even if a small mismatch arises. In practice, the problem of the mismatch possibly exists in many applications such as the application in mobile communication systems. This leads to many restrictions on the development of the conventional array beamforming.

A signal with cyclostationarity has the statistical property of correlating with either a frequency-shift or a complex-conjugate version of itself. By restoring this property at a known value of frequency separation, it is possible to favor the desired signal and to discriminate against the interference and noise [2], [5]. In addition, many cyclostationarity-exploiting algorithms avoid the need for training signals, the knowledge of array manifold, and noise characteristics. Moreover, adaptive beamforming can suppress not only Gaussian but non-Gaussian

Manuscript received December 28, 1998; revised February 15, 2000. This work was supported by the National Science Council under Grant NSC88-2218E002-027. The associate editor coordinating the review of this paper and approving it for publication was Prof. Dimitrios Hatzinakos.

The authors are with the Department of Electrical Engineering, National Taiwan University, Taipei, Taiwan, R.O.C.

Publisher Item Identifier S 1053-587X(00)04943-6. interferers as well by utilizing the signal cyclostationarity. As a result, adaptive beamforming utilizing signal cyclostationarity has been widely considered. Recently, a class of spectral self-coherent restoral (SCORE) algorithms has been presented in [6] to deal with the problem of blind adaptive signal extraction. When an infinite time-averaging interval is available, it has been shown in [6] that the solutions of the SCORE algorithms are the same as that of the conventional approaches developed based on maximizing the output signal-to-interference plus noise ratio (SINR) for adaptive beamforming. The a priori information that the SCORE algorithms require to work is only the cycle frequencies of the desired signal. Hence, its performance is sensitive to the accuracy of the presumed cycle frequencies. However, the actual cycle frequencies may not be known very well in some applications due, for example, to the phenomenon of Doppler shift. Therefore, it is worth evaluating the performance of the SCORE algorithms in the presence of cycle frequency error (CFE) and developing techniques to solve the problem. Some previous work considering the related problem in direction finding has been reported in [7]-[10]. A statistical analysis of the usual estimator of the cyclic autocorrelation is included in [7] and [8]. This analysis shows the effects of cycle leakage through a sinc window due to finite data samples. On the other hand, two approaches have been presented by [9] for dealing with the direction-finding problems due to CFE. The first one, called multicyclic MUSIC, uses a multicyclic correlation matrix to alleviate the performance degradation due to CFE. However, it is observed that this approach cannot tackle the problem effectively. The other one, which is called adaptive- $\alpha$ cyclic MUSIC, estimates the cycle frequency by using the FFT frequency estimator to compute the cyclic autocorrelation of the data received from a single sensor. The drawback of this approach is that huge memory is required for data storage to obtain satisfactory results. Variations of the adaptive- $\alpha$ cyclic MUSIC are explored in [10]. Based on the concept of subintervals, only the cyclic correlation matrices of the subintervals are required to reduce the memory requirements and the sensitivity of cyclic MUSIC to CFE. However, the techniques presented by [10] limit the cycle frequency coverage due to using subintervals.

In the literature, one of the SCORE algorithms presented by [6], namely, the least-square SCORE (LS-SCORE) algorithm, has been extensively considered [11]. In this paper, we first evaluate the performance of the LS-SCORE algorithm when the CFE regarding the desired signal exists. Using the theory of Fourier transform, the problem formulation for the LS-SCORE algorithm under CFE is presented. This results in an analytical formula that demonstrates the behavior of the performance degradation for the LS-SCORE algorithm. It is shown that 
the output SINR of an adaptive array beamformer using the LS-SCORE algorithm deteriorates like a sinc function as the number of data snapshots increases. Based on this theoretical result, we formulate an optimization problem to find an appropriate estimate of the cycle frequency of the desired signal iteratively. This estimate is then used to form an appropriate reference signal for the LS-SCORE algorithm. It is shown that adaptive array beamforming using the proposed method in conjunction with the LS-SCORE algorithm can effectively cure the problem of blind signal extraction in the presence of CFE.

This paper is organized as follows. In Section II, we briefly describe the original LS-SCORE algorithm presented by [6]. The performance analysis of the LS-SCORE algorithm in the presence of CFE is presented in Section III. An efficient method is then proposed to alleviate the performance degradation caused by the CFE in Section IV. The convergence analysis of the proposed method is presented in Section V. Several computer simulation examples for confirming the theoretical results and showing the effectiveness of the proposed method are provided in Section VI. Finally, we conclude the paper in Section VII.

\section{ADAPTIVE BEAMFORMING FOR CyClOSTATIONARY SignalS}

\section{A. Signal Cyclostationarity}

For a signal $s(t)$, its cyclic autocorrelation function and cyclic conjugate autocorrelation function are defined as the following infinite-time averages:

$$
R_{s}(\alpha, \tau)=\left\langle s\left(t+\frac{\tau}{2}\right) s^{*}\left(t-\frac{\tau}{2}\right) e^{-j 2 \pi \alpha t}\right\rangle_{\infty}
$$

and

$$
R_{s^{*}}(\alpha, \tau)=\left\langle s\left(t+\frac{\tau}{2}\right) s\left(t-\frac{\tau}{2}\right) e^{-j 2 \pi \alpha t}\right\rangle_{\infty}
$$

respectively, where the superscript "*" denotes the complex conjugate. $s(t)$ is then said to be cyclostationary if $R_{s}(\alpha, \tau)$ or $R_{s^{*}}(\alpha, \tau)$ does not equal zero at cycle frequency $\alpha$ for some time delay $\tau$. Many man-made communication signals exhibit cyclostationarity with cycle frequency equal to the twice of the carrier frequency or multiples of the baud rate or combinations of these [3], [4].

Let the data vector received by an array beamformer be designated as $\mathrm{x}(t)$. Then, its cyclic autocorrelation matrix and cyclic conjugate autocorrelation matrix are given by

$$
\mathbf{R}_{x x}(\alpha, \tau)=\left\langle\mathbf{x}\left(t+\frac{\tau}{2}\right) \mathbf{x}^{H}\left(t-\frac{\tau}{2}\right) e^{-j 2 \pi \alpha t}\right\rangle_{\infty}
$$

and

$$
\mathbf{R}_{x x^{\star}}(\alpha, \tau)=\left\langle\mathbf{x}\left(t+\frac{\tau}{2}\right) \mathbf{x}^{T}\left(t-\frac{\tau}{2}\right) e^{-j 2 \pi \alpha t}\right\rangle_{\infty}
$$

respectively, where the superscript " $H$ " denotes the conjugate transpose and " $T$ " the transpose. Next, we briefly describe adaptive beamforming utilizing signal cyclostationarity.

\section{B. LS-SCORE Algorithm}

Consider adaptive beamforming using an $M$-element antenna array excited by a signal of interest (SOI), $J$ interferers, and spatially white noise. The received data vector $\mathbf{x}(t)$ is then given by

$$
\mathbf{x}(t)=s(t) \mathbf{a}_{d}+\sum_{j=1}^{J} s_{j}(t) \mathbf{a}_{j}+\mathbf{n}(t)=s(t) \mathbf{a}_{d}+\mathbf{z}(t)
$$

where $s(t)$ and $s_{j}(t)$ denote the waveforms of the SOI and the $j$ th interferer, $\mathbf{a}_{d}$ and $\mathbf{a}_{j}$ the direction vectors of the SOI and the $j$ th interferer, and $\mathbf{n}(t)$ the noise vector, respectively. The array output is given by $y(t)=\mathbf{w}^{H} \mathbf{x}(t)$, where $\mathbf{w}$ denotes the weight vector.

Assume that $s(t)$ is cyclostationary and has a cycle frequency $\alpha$ but that $\mathbf{z}(t)$ is not cyclostationary at $\alpha$ and is temporally uncorrelated with $s(t)$. Based on the LS-SCORE algorithm of [6], a cost function is defined as

$$
F_{s c}(\mathbf{w} ; \mathbf{c})=\left\langle|y(t)-r(\alpha, t)|^{2}\right\rangle_{T}
$$

where the reference signal $r(\alpha, t)$ is given by

$$
r(\alpha, t)=\mathbf{c}^{H} \mathbf{x}^{*}(t-\tau) e^{j 2 \pi \alpha t}
$$

and $\langle\cdot\rangle_{T}$ denotes the average over the time interval $[0, T]$. $\mathbf{c}$ is a control vector and is fixed for the LS-SCORE algorithm. Let the sampling interval be $T_{s}$. The optimal weight vector $\mathbf{w}_{l s}$ minimizing (6) with $i$ data snapshots (i.e., $T=i T_{s}$ ) used is given by

$$
\hat{\mathbf{w}}_{l s}(\alpha, i)=\hat{\mathbf{R}}_{x x}^{-1}(i) \hat{\mathbf{r}}_{x r}(\alpha, i)
$$

where $\hat{\mathbf{R}}_{x x}(i)=\left\langle\mathbf{x}(t) \mathbf{x}^{H}(t)\right\rangle_{T}$ and $\hat{\mathbf{r}}_{x r}(\alpha, i)=\left\langle\mathbf{x}(t) r^{*}\right.$ $(\alpha, t)\rangle_{T}$ are the sample autocorrelation matrix of $\mathbf{x}(t)$ and the sample cross-correlation vector of $\mathrm{x}(t)$ and $r(\alpha, t)$ computed over $[0, T]$, respectively. For any control vector $\mathbf{c}$ as long as $\mathbf{c}^{T} \mathbf{a}_{d} \neq 0$, it is shown in [6] that (8) converges to the solution of conventional adaptive array beamforming based on the maximum output SINR criterion when $T$ approaches infinity.

\section{Performance Analysis in the Presence of CFE}

Here, the performance of the LS-SCORE algorithm in the presence of CFE is evaluated. We only present the results using the cyclic conjugate autocorrelation matrix for (8). Based on the presented results in this section, it is very straightforward to obtain the results using the cyclic autocorrelation matrix. Assume that the signal of interest $s(t)$, the interferers $s_{j}(t)$, and the vector noise $\mathbf{n}(t)$ are uncorrelated one another. From (4) and (5), the cyclic conjugate autocorrelation matrix at an arbitrary frequency $f$ can be written as

$$
\begin{aligned}
\mathbf{R}_{x x^{*}}(f, \tau)= & \left\langle\mathbf{x}\left(t+\frac{\tau}{2}\right) \mathbf{x}^{T}\left(t-\frac{\tau}{2}\right) e^{-j 2 \pi f t}\right\rangle_{\infty} \\
= & R_{s^{*}}(f, \tau) \mathbf{a}_{d} \mathbf{a}_{d}^{T}+\sum_{j=1}^{J} R_{s_{j}^{*}}(f, \tau) \mathbf{a}_{j} \mathbf{a}_{j}^{T} \\
& +\mathbf{R}_{n n^{*}}(f, \tau)
\end{aligned}
$$

where

$$
\begin{aligned}
& R_{s^{*}}(f, \tau)=\left\langle s\left(t+\frac{\tau}{2}\right) s\left(t-\frac{\tau}{2}\right) e^{-j 2 \pi f t}\right\rangle_{\infty} \\
& R_{s_{j}^{*}}(f, \tau)=\left\langle s_{j}\left(t+\frac{\tau}{2}\right) s_{j}\left(t-\frac{\tau}{2}\right) e^{-j 2 \pi f t}\right\rangle_{\infty}
\end{aligned}
$$


and

$$
\mathbf{R}_{n n^{*}}(f, \tau)=\left\langle\mathbf{n}\left(t+\frac{\tau}{2}\right) \mathbf{n}^{T}\left(t-\frac{\tau}{2}\right) e^{-j 2 \pi f t}\right\rangle_{\infty}
$$

Due to the fact that the cyclic spectrum of a cyclostationary signal is discrete in the cycle frequency, the cyclic conjugate autocorrelation function of the SOI can be written as

$$
R_{s^{*}}(f, \tau)=\sum_{n} d_{n}(\tau) \delta\left(f-\alpha_{n}\right)
$$

where

$$
\begin{array}{ll}
\alpha_{n} \text { 's } & \text { cycle frequencies of the SOI; } \\
d_{n}(\tau) & \text { corresponding strength at } \alpha_{n} ; \\
\delta(\cdot) & \text { Kronecker delta. }
\end{array}
$$

Next, we can rewrite the sample cross-correlation vector of $\mathbf{x}(t)$ and $r(\alpha, t)$ at $\alpha=f$ as

$$
\begin{aligned}
\hat{\mathrm{r}}_{x r}(f, i) & =\left\langle\mathrm{x}(t) r^{*}(f, t)\right\rangle_{T} \\
& =\frac{1}{T} \int_{-\infty}^{\infty} \operatorname{rect}\left(\frac{t}{T}\right) \mathrm{x}(t) r^{*}(f, t) d t
\end{aligned}
$$

where

$$
\operatorname{rect}\left(\frac{t}{T}\right)= \begin{cases}1, & \text { for }-\frac{T}{2} \leq t \leq \frac{T}{2} \\ 0, & \text { elsewhere }\end{cases}
$$

Substituting (7) into (14) yields

$$
\begin{aligned}
\hat{\mathbf{r}}_{x r}(f, i)= & \left\{\frac{1}{T} \int_{-\infty}^{\infty} \operatorname{rect}\left(\frac{t}{T}\right) \operatorname{rect}\left(\frac{t}{T}\right) \mathbf{x}(t) \mathbf{x}^{T}\right. \\
& \left.\cdot(t-\tau) e^{-2 \pi f t} d t\right\} \mathbf{c} .
\end{aligned}
$$

Utilizing the property of Fourier transform, we obtain

$$
\begin{aligned}
\hat{\mathbf{r}}_{x r}(f, i)= & {\left[\int_{-\infty}^{\infty} \operatorname{rect}\left(\frac{t}{T}\right) e^{-j 2 \pi f t} d t\right] } \\
& \star\left[\frac{1}{T} \int_{-\infty}^{\infty} \operatorname{rect}\left(\frac{t}{T}\right) \mathbf{x}(t) \mathbf{x}^{T}\right. \\
& \left.\cdot(t-\tau) e^{-j 2 \pi f t} d t\right] \mathbf{c} \\
= & T\left\{\operatorname{sinc}(f T) \star \hat{\mathbf{R}}_{x x^{\star}}(f, \tau) e^{-j \pi f \tau}\right\} \mathbf{c}
\end{aligned}
$$

where $\hat{\mathbf{R}}_{x x^{*}}(f, \tau)$ denotes the finite sample-size estimate of $\mathbf{R}_{x x^{\star}}(f, \tau)$, “*” the convolution operation, and

$$
\operatorname{sinc}(f T)=\frac{\sin (\pi f T)}{(\pi f T)} .
$$

From (9), we have

$$
\begin{aligned}
\hat{\mathbf{R}}_{x x^{*}}(f, \tau) e^{-j \pi f \tau} \mathbf{c} \approx & k_{d} \hat{R}_{s^{*}}(f, \tau) \mathbf{a}_{d} \\
& +\sum_{j=1}^{J} k_{j} \hat{R}_{s_{j}^{*}}(f, \tau) \mathbf{a}_{j}+\hat{\mathbf{k}}_{n}(f)
\end{aligned}
$$

where $k_{d}=\mathbf{a}_{d}^{T} \mathbf{c} e^{-j \pi f \tau}, k_{j}=\mathbf{a}_{j}^{T} \mathbf{c} e^{-j \pi f \tau}$, and $\hat{\mathbf{k}}_{n}(f)=\hat{\mathbf{R}}_{n n^{*}}$ $(f, \tau) \mathbf{c} e^{-j \pi f \tau}$. Moreover, the sample cyclic conjugate autocorrelation functions for the SOI and the $j$ th cyclostationary interferer are obtained from (13) as $\hat{R}_{s^{*}}(f, \tau) \approx$ $\Sigma_{n} \hat{d}_{n}(\tau) \operatorname{sinc}\left(\left(f-\alpha_{n}\right) T\right)$ and $\hat{R}_{s_{j}^{*}}(f, \tau) \approx \Sigma_{m} \hat{\rho}_{j, m}(\tau)$ sinc $\left(\left(f-\beta_{j, m}\right) T\right)$, respectively, where $\beta_{j, m}$ 's are the cycle frequencies of the $j$ th cyclostationary interferer, and $\hat{\rho}_{j, m}(\tau)$ 's are the corresponding strengths. Substituting (19) into (17) yields

$$
\begin{aligned}
\hat{\mathbf{r}}_{x r}(f, i) \approx & k_{d}\left\{\sum_{n} \hat{d}_{n}(\tau) \operatorname{sinc}\left(\left(f-\alpha_{n}\right) T\right)\right\} \mathbf{a}_{d} \\
& +\sum_{j, m} k_{j}\left\{\hat{\rho}_{j, m}(\tau) \operatorname{sinc}\left(\left(f-\beta_{j, m}\right) T\right)\right\} \mathbf{a}_{j} \\
& +T \tilde{\mathbf{k}}_{n}(f) \star \operatorname{sinc}(f T)
\end{aligned}
$$

where $T\{\operatorname{sinc}(f T)\} \star\left\{\operatorname{sinc}\left(\left(f-f_{0}\right) T\right)\right\}=\operatorname{sinc}\left(\left(f-f_{0}\right) T\right)$ for any constant $f_{0}$. Moreover, the interferers without cyclostationarity and noise are contained in $\tilde{\mathbf{k}}_{n}(f)$. In fact, $\hat{\mathbf{r}}_{x r}(f, i)$ also includes the sample cyclic cross correlations between the SOI and the interference, the SOI and noise, and the interference and noise. However, they are negligible when $T$ is large enough. Equation (20) shows the effects of cycle leakage through a sinc window due to finite data samples. These effects are also derived in [7] and [8] by using a different manner.

Next, let the presumed cycle frequency for the SOI be denoted by $\hat{\alpha}$, which differs from the cycle frequency $\alpha_{1}$ of the SOI by $\triangle \alpha$, where $\triangle \alpha$ represents the amount of CFE. Substituting $f=\alpha_{1}+\triangle \alpha$ into (20) yields

$$
\begin{aligned}
\hat{\mathbf{r}}_{x r}\left(\alpha_{1}+\Delta \alpha, i\right) \approx & k_{d}\left\{\hat{d}_{1}(\tau) \operatorname{sinc}(\triangle \alpha T)\right. \\
& \left.+\sum_{n \neq 1} \hat{d}_{n}(\tau) \operatorname{sinc}\left(\tilde{\alpha}_{n} T\right)\right\} \mathbf{a}_{d} \\
& +\sum_{j, m} k_{j}\left\{\hat{\rho}_{j, m}(\tau) \operatorname{sinc}\left(\tilde{\beta}_{j, m} T\right)\right\} \mathbf{a}_{j} \\
& +\left.T \tilde{\mathbf{k}}_{n}(f) \star \operatorname{sinc}(f T)\right|_{f=\alpha_{1}+\triangle \alpha}
\end{aligned}
$$

where $\tilde{\alpha}_{n}=\alpha_{1}-\alpha_{n}+\triangle \alpha$, and $\tilde{\beta}_{j, m}=\alpha_{1}-\beta_{j, m}+\triangle \alpha$. Consider the case where $\alpha_{1}$ and $\beta_{j, m}$ 's are well separated. Using the fact that the value of $\operatorname{sinc}(f T)$ is small enough for large $f T$, the effect of interference and noise is negligible when $\triangle \alpha=0$. Consequently, we note from (21) that $\hat{\mathbf{r}}_{x r}(f, i)$ is almost proportional to the direction vector $\mathbf{a}_{d}$. On the other hand, when $\triangle \alpha \neq 0$, due to the fact that $\operatorname{sinc}(\triangle \alpha T)=0$ when $T=T_{n}=$ $( \pm n / \triangle \alpha), n=1,2, \cdots$, we note that $\hat{\mathbf{r}}_{x r}(f, i)$ is approximately equal to $k_{d} \hat{d}_{1}(\tau) \operatorname{sinc}(\triangle \alpha T) \mathbf{a}_{d}$ if $T$ is far from $T_{n}$. However, when $T$ is close to $T_{n}$, the effect due to the interference and noise is not negligible. Thus, $\hat{\mathbf{r}}_{x r}(f, i)$ is no longer proportional to $\mathbf{a}_{d}$. This leads to performance degradation for the LS-SCORE algorithm. Owing to $T_{n}=( \pm n / \triangle \alpha)$, we would expect that there exist periodic nulls in the curves of the output SINR versus the number of snapshots for adaptive beamforming based on the LS-SCORE algorithm in the presence of CFE. Simulation results showing the performance degradation due to CFE are presented in Section VI for confirmation.

\section{PRoposed Method}

A main property of a signal that exhibits cyclostationarity is that spectrum lines are regenerated by quadratic nonlinear- 
ities only at discrete frequencies $\alpha_{n}$, as can be seen from (13). Based on (21), both of the $\operatorname{sinc}(\triangle \alpha T)$ and $\operatorname{sinc}\left(\tilde{\alpha}_{n} T\right)$ approach zero as $T$ increases. Hence, the performance degradation for the LS-SCORE algorithm becomes more severe as the number of snapshots increases. To alleviate this difficulty, we present an efficient method in conjunction with the LS-SCORE algorithm as follows.

From the optimal weight vector of (8), the total array output power becomes

$$
P_{l s}(\hat{\alpha}, i)=\hat{\mathbf{w}}_{l s}^{H}(\hat{\alpha}, i) \hat{\mathbf{R}}_{x x}(i) \hat{\mathbf{w}}_{l s}(\hat{\alpha}, i)
$$

for a given frequency $\hat{\alpha}$. Based on the analysis presented in Section III for the LS-SCORE algorithm, we note that the output power $P_{l s}(\hat{\alpha}, i)$ will show its maximum without CFE and decay to zero as the number of snapshots increases when CFE exists. Hence, an appropriate method for solving the problem due to CFE is to find the optimal $\hat{\alpha}$ that maximizes the total array output power $P_{l s}(\hat{\alpha}, i)$ in an appropriate neighborhood of $\alpha_{1}$, i.e., we resort to solving the following constrained optimization problem:

$$
\max _{\hat{\alpha}} \hat{\mathbf{w}}_{l s}^{H}(\hat{\alpha}, i) \hat{\mathbf{R}}_{x x}(i) \hat{\mathbf{w}}_{l s}(\hat{\alpha}, i) \quad \text { s.t. }|\hat{\alpha}-\hat{\alpha}(0)| \leq \zeta
$$

where $\hat{\alpha}(0)$ is the presumed cycle frequency, and $\zeta$ is a preset small number such that $\left|\alpha_{1}-\hat{\alpha}(0)\right| \leq \zeta$. Utilizing the conventional method of steepest descent for solving (23), we take the derivative of the objective function $P_{l s}(\hat{\alpha}, i)$ with respect to $\hat{\alpha}$. From (22), the derivative is given by

$$
\begin{aligned}
\vec{\alpha}_{\hat{\alpha}}\left(P_{l s}(\hat{\alpha}, i)\right) \equiv & \frac{\partial\left(\hat{\mathbf{w}}_{l s}^{H}(\hat{\alpha}, i) \hat{\mathbf{R}}_{x x}(i) \hat{\mathbf{w}}_{l s}(\hat{\alpha}, i)\right)}{\partial \hat{\alpha}} \\
= & \frac{\partial \hat{\mathbf{w}}_{l s}^{H}(\hat{\alpha}, i)}{\partial \hat{\alpha}} \hat{\mathbf{R}}_{x x}(i) \hat{\mathbf{w}}_{l s}(\hat{\alpha}, i) \\
& +\hat{\mathbf{w}}_{l s}^{H}(\hat{\alpha}, i) \hat{\mathbf{R}}_{x x}(i) \frac{\partial \hat{\mathbf{w}}_{l s}(\hat{\alpha}, i)}{\partial \hat{\alpha}} \\
= & 2 \times \operatorname{real}\left\{\frac{\partial \hat{\mathbf{w}}_{l s}^{H}(\hat{\alpha}, i)}{\partial \hat{\alpha}} \times\left(\hat{\mathbf{R}}_{x x}(i) \hat{\mathbf{w}}_{l s}(\hat{\alpha}, i)\right)\right\} \\
= & 2 \times \operatorname{real}\left\{\left(\mathbf{c}^{H} \hat{\mathbf{R}}_{x v}^{H}(\hat{\alpha}, i) \hat{\mathbf{R}}_{x x}^{-1}(i)\right)\right. \\
& \left.\times\left(\hat{\mathbf{R}}_{x x}(i) \hat{\mathbf{w}}_{l s}(\hat{\alpha}, i)\right)\right\} \\
= & 2 \times \operatorname{real}\left\{\mathbf{c}^{H} \hat{\mathbf{R}}_{x v}^{H}(\hat{\alpha}, i) \hat{\mathbf{w}}_{l s}(\hat{\alpha}, i)\right\}
\end{aligned}
$$

where real $\{x\}$ denotes the real part of $x$, and $\hat{\mathbf{R}}_{x v}(\hat{\alpha}, i)$ is given by

$$
\hat{\mathbf{R}}_{x v}(\hat{\alpha}, i)=\left\langle\mathbf{x}(t) \mathbf{x}^{T}(t-\tau)(-j 2 \pi t) e^{-2 j \pi \hat{\alpha} t}\right\rangle_{T}
$$

where $\langle\cdot\rangle_{T}=(1 / T) \int_{-(T / 2)}^{T / 2} \cdot d t$. We note that since the array output power reaches the local maximum at $\alpha_{1}$, it should be approximately a concave function in an appropriate neighborhood of $\alpha_{1}$. Therefore, the derivatives used in the solution of (23) will lead to maximization of (23). Accordingly, the updated value of the estimate for the cycle frequency $\alpha$ at the time instant $(i+1)$ is computed by using the following simple recursive formula:

$$
\hat{\alpha}(i+1)=\hat{\alpha}(i)+\left.\mu(i) \underset{\hat{\alpha}}{\Delta}\left(P_{l_{s}}(\hat{\alpha}, i)\right)\right|_{\hat{\alpha}=\hat{\alpha}(i)}
$$

where $\mu(i)$ is a positive real-valued parameter referred to as the step-size parameter. Examining the derivative given by (24), we set the step-size parameter $\mu(i)$ equal to

$$
\mu(i)=\frac{1}{\left\|\hat{\mathbf{R}}_{x v}(\hat{\alpha}(i), i)\right\|^{q}}
$$

to ensure the convergence of the steepest-descent algorithm used by (26), where $\|\mathbf{A}\|$ denotes the maximum singular value of the matrix $\mathbf{A}$, and $q$ is an appropriate positive real value determined by experiment. The updated value of the weight vector at the time instant $(i+1)$ is obtained by substituting (26) into (8) and given by

$$
\hat{\mathbf{w}}_{l s}(\hat{\alpha}(i+1), i+1)=\hat{\mathbf{R}}_{x x}^{-1}(i+1) \hat{\mathbf{r}}_{x r}(\hat{\alpha}(i+1), i+1) .
$$

For practical implementation, we compute the required sample data correlation matrix $\hat{\mathbf{R}}_{x x}(i), \hat{\mathbf{R}}_{x v}(\hat{\alpha}(i), i)$, and the cross-correlation vector $\hat{\mathbf{r}}_{x r}(\hat{\alpha}(i), i)$ by utilizing (29), shown at the bottom of the page, where $\mathrm{x}(i)=\mathbf{x}\left(i T_{s}\right)$, $r(\hat{\alpha}(i), k)=\mathbf{c}^{H} \mathbf{x}^{*}(k) e^{j 2 \pi \hat{\alpha}(i) k T_{s}}$, and $L_{b}$ is a preset positive integer. Since $\hat{\alpha}(i)$ becomes more appropriate as $i$ increases as noted from (29), we use $L_{b}$ data samples to update the corresponding correlations for increasing the effect of $\hat{\alpha}(i)$ on the estimates of the considered correlations shown by (29).

Consider the required computational complexity. From (24) and (29), it is easy to show that the computational complexity for obtaining $\Delta_{\hat{\alpha}}\left(P_{l s}(\hat{\alpha}, i)\right)$ is $O\left(M^{2}\right)$, where $M$ is the number of sensor elements. Hence, from (26), the computational complexity required to estimate the cycle frequency is $O\left(M^{2}\right)$ for the proposed method. Moreover, the proposed method does not require a searching procedure like the simple fast Fourier

$$
\begin{aligned}
\hat{\mathbf{R}}_{x x}(i) & =\left(1-\frac{1}{i}\right) \hat{\mathbf{R}}_{x x}(i-1)+\frac{1}{i} \mathbf{x}(i) \mathbf{x}^{H}(i) \\
\hat{\mathbf{R}}_{x v}(\hat{\alpha}(i), i) & = \begin{cases}\frac{1}{i} \sum_{k=1}^{i} \mathbf{x}(k) \mathbf{x}^{T}(k) e^{-j 2 \pi \hat{\alpha}(i) k T_{s}}\left(-j 2 \pi k T_{s}\right), & \text { if } i \leq L_{b} \\
\left(1-\frac{1}{i}\right) \hat{\mathbf{R}}_{x v}(\hat{\alpha}(i-1), i-1)+\frac{1}{i} \sum_{k=i-L_{b}+1}^{i} \mathbf{x}(k) \mathbf{x}^{T}(k) e^{-j 2 \pi \hat{\alpha}(i) k T_{s}}\left(-j 2 \pi k T_{s}\right), & \text { if } i>L_{b}\end{cases} \\
\hat{\mathbf{r}}_{x r}(\hat{\alpha}(i), i) & = \begin{cases}\frac{1}{i} \sum_{k=1}^{i} \mathbf{x}(k) r^{*}(\hat{\alpha}(i), k), & \text { if } i \leq L_{b} \\
\left(1-\frac{1}{i}\right) \hat{\mathbf{r}}_{x r}(\hat{\alpha}(i-1), i-1)+\frac{1}{i} \sum_{k=i-L_{b}+1}^{i} \mathbf{x}(k) r^{*}(\hat{\alpha}(i), k), & \text { if } i>L_{b} .\end{cases}
\end{aligned}
$$


transform (FFT) frequency estimator used in the adaptive- $\alpha$ cyclic MUSIC [9]. This searching procedure requires too many computations at those frequencies other than the true cycle frequency. As for nonadaptive methods, such as the multicyclic MUSIC presented in [9], it has been shown in [9] that the interference and noise contribution is increased by using a multicyclic correlation matrix. Further, the multicyclic MUSIC may lead to severe performance degradation when none of the presumed cycle frequencies equals the true one.

\section{Convergence of the Proposed Method}

In this section, the convergence property of the proposed method is evaluated. For simplicity, we set $\tau=0$. The array output power can be rewritten as

$$
\begin{aligned}
P_{l s}(\hat{\alpha}, i)= & \mathbf{w}_{l s}^{H}(\hat{\alpha}, i) \hat{\mathbf{R}}_{x x}(i) \mathbf{w}_{l s}(\hat{\alpha}, i) \\
= & \hat{\mathbf{r}}_{x r}^{H}(\hat{\alpha}, i) \hat{\mathbf{R}}_{x x}^{-1}(i) \hat{\mathbf{r}}_{x r}(\hat{\alpha}, i) \\
= & \mathbf{c}^{H} \hat{\mathbf{R}}_{x x^{*}}^{H}(\hat{\alpha}, i) \hat{\mathbf{R}}_{x x}^{-1}(i) \hat{\mathbf{R}}_{x x^{*}}(\hat{\alpha}, i) \mathbf{c} \\
= & \frac{1}{i^{2}} \sum_{l=0}^{i-1} \sum_{k=0}^{i-1}\left\{\mathbf{c}^{H} \mathbf{x}^{*}(l) \mathbf{x}^{T}(k) \mathbf{c}\right\} \\
& \cdot\left\{\mathbf{x}^{H}(l) \hat{\mathbf{R}}_{x x}^{-1}(i) \mathbf{x}(k)\right\} e^{-j 2 \pi \hat{\alpha}\left\{(k-l) T_{s}\right\}} .
\end{aligned}
$$

Using (5), we can express the term $\mathbf{x}^{H}(l) \hat{\mathbf{R}}_{x x}^{-1}(i) \mathbf{x}(k)$ in (30) as

$$
\begin{aligned}
\mathbf{x}^{H}(l) & \hat{\mathbf{R}}_{x x}^{-1}(i) \mathbf{x}(k) \\
\approx & s^{*}(l) s(k)\left\{\mathbf{a}_{d}^{H} \hat{\mathbf{R}}_{x x}^{-1}(i) \mathbf{a}_{d}\right\} \\
& +\sum_{j=1}^{J} s_{j}^{*}(l) s_{j}(k)\left\{\mathbf{a}_{j}^{H} \hat{\mathbf{R}}_{x x}^{-1}(i) \mathbf{a}_{j}\right\} \\
& + \text { noise-related terms. }
\end{aligned}
$$

The approximation is obtained due to $\mathbf{a}_{d}^{H} \hat{\mathbf{R}}_{x x}^{-1}(i) \mathbf{a}_{j} \approx 0$ and $\mathbf{a}_{j}^{H} \hat{\mathbf{R}}_{x x}^{-1}(i) \mathbf{a}_{\bar{j}} \approx 0$ as the number of data snapshots $i$ approaches infinite, where $j, \bar{j}=1,2, \cdots, J$ and $j \neq \bar{j}$. For the term $\mathbf{c}^{H} \mathbf{x}^{*}(l) \mathbf{x}^{T}(k) \mathbf{c}$, we have

$$
\begin{aligned}
\mathbf{c}^{H} \mathbf{x}^{*}(l) & \mathbf{x}^{T}(k) \mathbf{c} \\
= & \mathbf{c}^{H}\left\{s(l) \mathbf{a}_{d}+\sum_{j=1}^{J} s_{j}(l) \mathbf{a}_{j}+\mathbf{n}(l)\right\}^{*}\left\{s(k) \mathbf{a}_{d}\right. \\
& \left.+\sum_{j=1}^{J} s_{j}(k) \mathbf{a}_{j}+\mathbf{n}(k)\right\}^{T} \mathbf{c} \\
= & s^{*}(l) s(k)\left\{\mathbf{c}^{H} \mathbf{a}_{d}^{*} \mathbf{a}_{d}^{T} \mathbf{c}\right\}+\sum_{j=1}^{J} s_{j}^{*}(l) s_{j}(k)\left\{\mathbf{c}^{H} \mathbf{a}_{j}^{*} \mathbf{a}_{j}^{T} \mathbf{c}\right\} \\
& +\mathbf{c}^{H} \mathbf{n}^{*}(l) \mathbf{n}^{T}(k) \mathbf{c}+\text { cross terms. }
\end{aligned}
$$

It follows from (31) and (32) that

$$
\begin{aligned}
&\left\{\mathbf{c}^{H} \mathbf{x}^{*}(l) \mathbf{x}^{T}(k) \mathbf{c}\right\}\left\{\mathbf{x}^{H}(l) \hat{\mathbf{R}}_{x x}^{-1}(i) \mathbf{x}(k)\right\} \\
& \approx s^{*}(l) s^{*}(l) s(k) s(k)\left\{\mathbf{a}_{d}^{H} \hat{\mathbf{R}}_{x x}^{-1}(i) \mathbf{a}_{d}\right\}\left\{\mathbf{c}^{H} \mathbf{a}_{d}^{*} \mathbf{a}_{d}^{T} \mathbf{c}\right\} \\
& \quad+\sum_{j=1}^{J} s_{j}^{*}(l) s_{j}^{*}(l) s_{j}(k) s_{j}(k)\left\{\mathbf{a}_{j}^{H} \hat{\mathbf{R}}_{x x}^{-1}(n) \mathbf{a}_{j}\right\} \\
& \quad \cdot\left\{\mathbf{c}^{H} \mathbf{a}_{j}^{*} \mathbf{a}_{j}^{T} \mathbf{c}\right\}
\end{aligned}
$$$$
+ \text { the cross terms including noise and interference. }
$$

Then, substituting (33) into (30) and performing some necessary algebraic manipulations, we can obtain the following approximation as the number of data snapshots $i$ approaches infinity:

$$
\begin{aligned}
P_{l s}(\hat{\alpha}, i)= & \mathbf{w}_{l s}^{H}(\hat{\alpha}, i) \hat{\mathbf{R}}_{x x}(i) \mathbf{w}_{l s}(\hat{\alpha}, i) \\
\approx & \left\{\frac{1}{i^{2}} \sum_{l=0}^{i-1} \sum_{k=0}^{i-1}\left\{s(l) s(l) e^{-j 2 \pi \hat{\alpha} l T_{s}}\right\}^{*}\right. \\
& \left.\cdot\left\{s(k) s(k) e^{-j 2 \pi \hat{\alpha} k T_{s}}\right\}\right\}\left\{\mathbf{a}_{d}^{H} \hat{\mathbf{R}}_{x x}^{-1}(i) \mathbf{a}_{d}\right\} \\
& \cdot\left\{\mathbf{c}^{H} \mathbf{a}_{d}^{*} \mathbf{a}_{d}^{T} \mathbf{c}\right\} \\
& +\sum_{j=1}^{J}\left\{\frac{1}{i^{2}} \sum_{l=0}^{i-1} \sum_{k=0}^{i-1}\left\{s_{j}(l) s_{j}(l) e^{-j 2 \pi \hat{\alpha} l T_{s}}\right\}^{*}\right. \\
& \cdot\left\{s_{j}(k) s_{j}(k) e^{-j 2 \pi \hat{\alpha} k T_{s}}\right\}\left\{\mathbf{a}_{j}^{H} \hat{\mathbf{R}}_{x x}^{-1}(i) \mathbf{a}_{j}\right\} \\
& \left.\cdot\left\{\mathbf{c}^{H} \mathbf{a}_{j}^{*} \mathbf{a}_{j}^{T} \mathbf{c}\right\}\right\} \\
=\mid & \left|\hat{R}_{s^{*}}(\hat{\alpha}, 0)\right|^{2}\left\{\mathbf{a}_{d}^{H} \hat{\mathbf{R}}_{x x}^{-1}(i) \mathbf{a}_{d}\right\}\left\{\mathbf{c}^{H} \mathbf{a}_{d}^{*} \mathbf{a}_{d}^{T} \mathbf{c}\right\} \\
& +\sum_{j=1}^{J}\left|\hat{R}_{s_{j}^{*}}(\hat{\alpha}, 0)\right|^{2}\left\{\mathbf{a}_{j}^{H} \hat{\mathbf{R}}_{x x}^{-1}(i) \mathbf{a}_{j}\right\}\left\{\mathbf{c}^{H} \mathbf{a}_{j}^{*} \mathbf{a}_{j}^{T} \mathbf{c}\right\} .
\end{aligned}
$$

We note from (34) that the cross terms disappear due to the stationarity of $\mathbf{n}(t)$ and the assumed uncorrelation among $s(t)$, the $s_{j}(t)$ 's, and $\mathbf{n}(t)$. Based on the result given by (34), we can rewrite (24) as

$$
\begin{aligned}
\vec{\alpha}_{\hat{\alpha}}\left(P_{l s}(\hat{\alpha}, i)\right) \approx & \frac{\partial\left|\hat{R}_{s^{*}}(\hat{\alpha}, 0)\right|^{2}}{\partial \hat{\alpha}}\left\{\mathbf{a}_{d}^{H} \hat{\mathbf{R}}_{x x}^{-1}(i) \mathbf{a}_{d}\right\}\left\{\mathbf{c}^{H} \mathbf{a}_{d}^{*} \mathbf{a}_{d}^{T} \mathbf{c}\right\} \\
& +\sum_{j=1}^{J} \frac{\partial\left|\hat{R}_{s_{j}^{*}}(\hat{\alpha}, 0)\right|^{2}}{\partial \hat{\alpha}}\left\{\mathbf{a}_{j}^{H} \hat{\mathbf{R}}_{x x}^{-1}(i) \mathbf{a}_{j}\right\} \\
& \cdot\left\{\mathbf{c}^{H} \mathbf{a}_{j}^{*} \mathbf{a}_{j}^{T} \mathbf{c}\right\} .
\end{aligned}
$$

For BPSK signals, we assume that

$$
s(t)=A_{s} e^{j\left(\pi \alpha t+\Phi_{s}(t)\right)} \quad \text { and } \quad s_{j}(t)=A_{j} e^{j\left(\pi \beta_{j} t+\Phi_{j}(t)\right)}
$$

where $A_{s}$ and $A_{j}$ are the constant amplitudes, and $\Phi_{s}(t)$ and $\Phi_{j}(t)$ represent the random phases equal to $\pm(\pi / 2)$ for the desired signal and the $j$ th interferer, respectively. Accordingly, we have

$$
\begin{aligned}
& \hat{R}_{s^{*}}(\hat{\alpha}, 0)=-A_{s}^{2} \operatorname{sinc}((\hat{\alpha}-\alpha) T) \quad \text { and } \\
& \hat{R}_{s_{j}^{*}}(\hat{\alpha}, 0)=-A_{j}^{2} \operatorname{sinc}\left(\left(\hat{\alpha}-\beta_{j}\right) T\right) .
\end{aligned}
$$

Equation (37) leads to

$$
\begin{aligned}
& \frac{\partial\left|\hat{R}_{s^{*}}(\hat{\alpha}, 0)\right|^{2}}{\partial \hat{\alpha}}=2 A_{s}^{4} \operatorname{sinc}((\hat{\alpha}-\alpha) T) \frac{\partial \operatorname{sinc}((\hat{\alpha}-\alpha) T)}{\partial \hat{\alpha}} \text { and } \\
& \frac{\partial\left|\hat{R}_{s_{j}^{*}}(\hat{\alpha}, 0)\right|^{2}}{\partial \hat{\alpha}}=2 A_{j}^{4} \operatorname{sinc}\left(\left(\hat{\alpha}-\beta_{j}\right) T\right) \frac{\partial \operatorname{sinc}\left(\left(\hat{\alpha}-\beta_{j}\right) T\right)}{\partial \hat{\alpha}} .
\end{aligned}
$$

Next, consider the case of $\hat{\alpha}=\alpha+\Delta \alpha$, and set $\alpha+\Delta \alpha-\beta_{j}=$ $\Delta \beta_{j}$. Then, (35) becomes

$$
\begin{aligned}
\Delta_{\hat{\alpha}}\left(P_{l s}(\hat{\alpha}, i)\right)= & 2 \frac{\partial \operatorname{sinc}(\Delta \alpha T)}{\partial \Delta \alpha} A_{s}^{4} \operatorname{sinc}(\Delta \alpha T) \\
& \cdot\left\{\mathbf{a}_{d}^{H} \hat{\mathbf{R}}_{x x}^{-1}(i) \mathbf{a}_{d}\right\}\left\{\mathbf{c}^{H} \mathbf{a}_{d}^{*} \mathbf{a}_{d}^{T} \mathbf{c}\right\} \\
& +2 \sum_{j=1}^{J} \frac{\partial \operatorname{sinc}\left(\Delta \beta_{j} T\right)}{\partial \Delta \beta_{j}} A_{j}^{4} \operatorname{sinc}\left(\Delta \beta_{j} T\right) \\
& \cdot\left\{\mathbf{a}_{j}^{H} \hat{\mathbf{R}}_{x x}^{-1}(i) \mathbf{a}_{j}\right\}\left\{\mathbf{c}^{H} \mathbf{a}_{j}^{*} \mathbf{a}_{j}^{T} \mathbf{c}\right\} .
\end{aligned}
$$


To show the influence of the choice of the starting point in (25), we redefine the finite-time average of (25) as $\langle\cdot\rangle_{T}=(1 / T) \int_{t_{0}}^{t_{0}+T} \cdot d t$. Then, substituting (36) into (25) and performing some algebraic manipulations, we obtain

$$
\begin{aligned}
\hat{\mathbf{R}}_{x v}(\alpha, i) & \left\langle\left[s(t) \mathbf{a}_{d}+\sum_{j=1}^{J} s_{j}(t) \mathbf{a}_{j}+\mathbf{n}(t)\right]\right. \\
= & {\left.\left[j s(t) \mathbf{a}_{d}+\sum_{j=1}^{J} s_{j}(t) \mathbf{a}_{j}+\mathbf{n}(t)\right]^{T} e^{-j 2 \pi \hat{\alpha} t}(-j 2 \pi t)\right\rangle } \\
\approx & \left\langle-j 2 \pi t s^{2}(t) e^{-j 2 \pi \hat{\alpha} t}\right\rangle_{T} \mathbf{a}_{d} \mathbf{a}_{d}^{T} \\
& +\sum_{j=1}^{J}\left\langle-j 2 \pi t s_{j}^{2}(t) e^{-j 2 \pi \hat{\alpha} t}\right\rangle_{T} \mathbf{a}_{j} \mathbf{a}_{j}^{T} \\
= & \frac{A_{s}^{2} e^{-j 2 \pi \Delta \alpha\left(t_{0}+(T / 2)\right)}}{\pi \Delta \alpha^{2} T} \\
& \cdot\{\sin (\pi \Delta \alpha T)-\pi \Delta \alpha T \cos (\pi \Delta \alpha T) \\
& \left.+j \pi \Delta \alpha\left(2 t_{0}+T\right) \sin (\pi \Delta \alpha T)\right\} \mathbf{a}_{d} \mathbf{a}_{d}^{T} \\
& +\sum_{j=1}^{J} \frac{A_{j}^{2} e^{-j 2 \pi \Delta \beta_{j}\left(t_{0}+(T / 2)\right)}}{\pi \Delta \beta_{j}^{2} T} \\
& \cdot\left\{\sin \left(\pi \Delta \beta_{j} T\right)-\pi \Delta \beta_{j} T \cos \left(\pi \Delta \beta_{j} T\right)\right. \\
& \left.+j \pi \Delta \beta_{j}\left(2 t_{0}+T\right) \sin \left(\pi \Delta \beta_{j} T\right)\right\} \mathbf{a}_{j} \mathbf{a}_{j}^{T} .
\end{aligned}
$$

At the time instant $i$, the time interval $T=i T_{s}$ and the estimated cycle frequency is given by $\hat{\alpha}(i)$. In order to make the influence due to interference negligible, it is appropriate to make sure that the relationship given by

$$
\left|\Delta \alpha(i)\left\{i T_{s}\right\}\right| \leq \frac{1}{2}
$$

is kept, where $\Delta \alpha(i)=\hat{\alpha}(i)-\alpha$. Accordingly, (39) becomes

$$
\begin{aligned}
{\underset{\hat{\alpha}}{\Delta}(}_{\left.P_{l s}(\hat{\alpha}, i)\right)\left.\right|_{\hat{\alpha}=\hat{\alpha}(i)}}^{\approx} & \left.2 \frac{\partial \operatorname{sinc}(\Delta \alpha T)}{\partial \Delta \alpha}\right|_{\Delta \alpha=\Delta \alpha(i)} A_{s}^{4} \operatorname{sinc}(\Delta \alpha(i) T) \\
& \cdot\left\{\mathbf{a}_{d}^{H} \hat{\mathbf{R}}_{x x}^{-1}(i) \mathbf{a}_{d}\right\}\left\{\mathbf{c}^{H} \mathbf{a}_{d}^{*} \mathbf{a}_{d}^{T} \mathbf{c}\right\} \\
= & 2 \frac{\pi \Delta \alpha(i) T \cos (\pi \Delta \alpha(i) T)-\sin (\pi \Delta \alpha(i) T)}{\pi \Delta \alpha^{2}(i) T} \\
& \cdot A_{s}^{4} \operatorname{sinc}(\Delta \alpha(i) T)\left\{\mathbf{a}_{d}^{H} \hat{\mathbf{R}}_{x x}^{-1}(i) \mathbf{a}_{d}\right\}\left\{\mathbf{c}^{H} \mathbf{a}_{d}^{*} \mathbf{a}_{d}^{T} \mathbf{c}\right\}
\end{aligned}
$$

and (40) becomes

$$
\begin{aligned}
\hat{\mathbf{R}}_{x v}(\hat{\alpha}(i), i) \approx & \frac{A_{s}^{2} e^{-j 2 \pi \Delta \alpha(i)\left(t_{0}+(T / 2)\right)}}{\pi \Delta \alpha^{2}(i) T} \\
& \cdot\{\sin (\pi \Delta \alpha(i) T)-\pi \Delta \alpha(i) T \cos (\pi \Delta \alpha(i) T) \\
& \left.+j \pi \Delta \alpha(i)\left(2 t_{0}+T\right) \sin (\pi \Delta \alpha(i) T)\right\} \mathbf{a}_{d} \mathbf{a}_{d}^{T} .
\end{aligned}
$$

Equation (43) reveals that the maximum singular value of $\hat{\mathbf{R}}_{x v}(\hat{\alpha}(i), i)$ is approximately equal to

$$
\begin{aligned}
& \frac{M^{2} A_{s}^{2}}{\pi \Delta \alpha^{2}(i) T} \mid \sin (\pi \Delta \alpha(i) T)-\pi \Delta \alpha(i) T \cos (\pi \Delta \alpha(i) T) \\
& \quad+j \pi \Delta \alpha(i)\left(2 t_{0}+T\right) \sin (\pi \Delta \alpha(i) T) \mid
\end{aligned}
$$

where $\mathbf{a}_{d}^{H} \mathbf{a}_{d}=M$. As a result, the step-size parameter of (27) is given by

$$
\begin{aligned}
\mu(i)= & \left\{\frac{M^{2} A_{s}^{2}}{\pi \Delta \alpha^{2}(i) T} \mid \sin (\pi \Delta \alpha(i) T)\right. \\
& -\pi \Delta \alpha(i) T \cos (\pi \Delta \alpha(i) T) \\
& \left.+j \pi \Delta \alpha(i)\left(2 t_{0}+T\right) \sin (\pi \Delta \alpha(i) T) \mid\right\}^{-q} .
\end{aligned}
$$

Based on the results of (42) and (44), we will prove that $\left|\Delta \alpha(i+1)\left\{(i+1) T_{s}\right\}\right| \leq \frac{1}{2}$ if $\left|\Delta \alpha(i)\left\{i T_{s}\right\}\right| \leq \frac{1}{2}$ to ensure the convergence of the proposed method. It follows from (26) that

$$
\Delta \alpha(i+1)=\Delta \alpha(i)+\left.\mu(i) \underset{\hat{\alpha}}{\Delta}\left(P_{l s}(\hat{\alpha}, i)\right)\right|_{\hat{\alpha}=\hat{\alpha}(i)} .
$$

Therefore, we have

$$
\begin{aligned}
& \Delta \alpha(i+1)\left\{(i+1) T_{s}\right\} \\
&=\left\{\Delta \alpha(i)+\mu(i){ }_{\hat{\alpha}}\left(P_{l s}(\hat{\alpha}(i), i)\right)\right\}\left\{(i+1) T_{s}\right\} \\
& \approx\left\{\Delta \alpha(i)+2 M^{-2 q} A_{s}^{4-2 q}\left\{\mathbf{a}_{d}^{H} \hat{\mathbf{R}}_{x x}^{-1}(i) \mathbf{a}_{d}\right\}\right. \\
& \cdot\left\{\mathbf{c}^{H} \mathbf{a}_{d}^{*} \mathbf{a}_{d}^{T} \mathbf{c}\right\} \operatorname{sinc}(\Delta \alpha(i) T) \\
& \cdot \frac{\pi \Delta \alpha(i) T \cos (\pi \Delta \alpha(i) T)-\sin (\pi \Delta \alpha(i) T)}{\left(\pi \Delta \alpha^{2}(i) T\right)^{1-q}} \\
& \cdot \mid \sin (\pi \Delta \alpha(i) T)-\pi \Delta \alpha(i) T \cos (\pi \Delta \alpha(i) T) \\
&\left.+\left.j \pi \Delta \alpha(i)\left(2 t_{0}+T\right) \sin (\pi \Delta \alpha(i) T)\right|^{-q}\right\}\left.\right|_{T=i T_{s}} \\
& \cdot\left\{(i+1) T_{s}\right\} .
\end{aligned}
$$

Substituting the extreme value $\frac{1}{2}$ for $\Delta \alpha(i)\left\{i T_{s}\right\}$ into (46) and performing some necessary algebraic manipulations yields

$$
\begin{aligned}
\Delta \alpha(i+1) & \left\{(i+1) T_{s}\right\} \\
\approx & \frac{1}{2}+\frac{1}{2 i}-\left\{2^{4-2 q} \pi^{q-2} M^{-2 q} A_{s}^{4-2 q}\left\{\mathbf{a}_{d}^{H} \hat{\mathbf{R}}_{x x}^{-1}(i) \mathbf{a}_{d}\right\}\right. \\
& \left.\cdot\left\{\mathbf{c}^{H} \mathbf{a}_{d}^{*} \mathbf{a}_{d}^{T} \mathbf{c}\right\} T_{s}^{2-q}\right\}\left|1+j \frac{\pi}{2}\left(1+\frac{2 t_{0}}{i T_{s}}\right)\right|^{-q} \frac{i+1}{i^{q-1}} .
\end{aligned}
$$

When $i$ is large enough, we have, from (47), the following.

1) If $t_{0}=-(T / 2)=-\left(i T_{s} / 2\right)$, then, we have the expression at the bottom of the page, where $C_{1}$ is always non-negative and approximately independent of $i$ by neglecting the effect due to finite data samples after $i$ is large enough.

$$
\Delta \alpha(i+1)\left\{(i+1) T_{s}\right\} \approx \frac{1}{2}+\frac{1}{2 i}-\underbrace{\left\{2^{4-2 q} \pi^{q-2} M^{-2 q} A_{s}^{4-2 q}\left\{\mathbf{a}_{d}^{H} \hat{\mathbf{R}}_{x x}^{-1}(i) \mathbf{a}_{d}\right\}\left\{\mathbf{c}^{H} \mathbf{a}_{d}^{*} \mathbf{a}_{d}^{T} \mathbf{c}\right\} T_{s}^{2-q}\right\}}_{C_{1}} \frac{i+1}{i^{q-1}}
$$


2) If $t_{0}$ is a constant, say, $t_{0}=0$, then we have the first expression at the bottom of the page, where

$$
\left|1+j \frac{\pi}{2}\left(1+\frac{2 t_{0}}{i T_{s}}\right)\right|^{-q} \approx\left(1+\frac{\pi^{2}}{4}\right)^{-q / 2}
$$

and $C_{2}$ is always non-negative and independent of $i$ when $i$ is large enough.

Hence, under the condition of $\left|\Delta \alpha(i)\left\{i T_{s}\right\}\right| \leq \frac{1}{2}$, we have

$$
-1 \leq \frac{1}{2(i-1)}-C \frac{i}{(i-1)^{q-1}} \leq 0
$$

where $C=C_{1}$ and $C_{2}$ when $t_{0}$ equals $-T / 2$ and a constant, respectively. It follows from (48) that

$$
\begin{aligned}
\frac{1}{2 i}- & \frac{(2 i-1)(i+1)}{2 i^{q}(i-1)^{2-q}} \\
& \leq \frac{1}{2 i}-C \frac{i+1}{i^{q-1}} \leq \frac{1}{2 i}-\frac{i+1}{2 i^{q}(i-1)^{2-q}} .
\end{aligned}
$$

Consider the situation that $1 \leq q \leq 2$. This leads to

$$
\begin{aligned}
\frac{1}{2 i}- & \frac{i+1}{2 i^{q}(i-1)^{2-q}} \\
& =\frac{1}{2 i}\left[1-\left(\frac{i+1}{i}\right)^{q-1}\left(\frac{i+1}{i-1}\right)^{2-q}\right] \leq 0
\end{aligned}
$$

and

$$
\frac{1}{2 i}-\frac{(2 i-1)(i+1)}{2 i^{q}(i-1)^{2-q}}>-1 \quad \text { if } q=2 \text { and }<-1 \text { if } q=1 .
$$

Therefore, we can see from (47) that there exists some $q$ between 1 and 2 such that $\left|\Delta \alpha(i+1)\left\{(i+1) T_{s}\right\}\right| \leq \frac{1}{2}$, irrespective of $t_{0}$. Similarly, substituting the other extreme value $-1 / 2$ for $\Delta \alpha(i)\left\{i T_{s}\right\}$ into (46) and performing some necessary algebraic manipulations yields (50), which is the second expression at the bottom of the page, when $i$ is large enough. Following the same procedure as described above and letting $C=C_{1}$ and $C_{2}$ when $t_{0}$ equals $-(T / 2)$ and a constant, respectively, it is easy to show that

$$
\begin{aligned}
1 \geq & -\frac{1}{2 i}+\frac{(2 i-1)(i+1)}{2 i^{q}(i-1)^{2-q} \geq-\frac{1}{2 i}} \\
& C \frac{i+1}{i^{q-1}} \geq-\frac{1}{2 i}+\frac{i+1}{2 i^{q}(i-1)^{2-q}} \geq 0
\end{aligned}
$$

for some $q$ with value between 1 and 2 . Consequently, there exists some $q$ that can be appropriately chosen between 1 and 2 to ensure that $\left|\Delta \alpha(i+1)\left\{(i+1) T_{s}\right\}\right| \leq \frac{1}{2}$ if $\left|\Delta \alpha(l)\left\{l T_{s}\right\}\right| \leq \frac{1}{2}$ for all $l \leq i$, irrespective of the choice of the starting point $t_{0}$.

\section{COMPUTER SimUlation EXAMPLES}

In this section, several simulation examples performed on a Pentium II-300 PC using MATLAB programming language are presented to confirm the theoretical results and show the effectiveness of the proposed method. For all simulation examples, we have a uniform linear array with number of elements $M=21$ and interelement spacing $=\lambda / 2$, where $\lambda$ is the wavelength of the SOI that is BPSK with rectangular pulse shape and impinging on the array from the broadside. The SOI has cycle frequency $\alpha_{1}=2$, baud rate $=5 / 11$, and signal-to-noise ratio $=5 \mathrm{~dB}$. Two interferers are also rectangular pulse shaped BPSK signals having cycle frequencies equal to 4.6 and 7.8 and impinging on the array from $30^{\circ}$ and $40^{\circ}$ off broadside, respectively. Moreover, the interferers have the interference-to-noise ratio and baud rate equal to $10 \mathrm{~dB}$ and 5/11, respectively. The noise received by the array is spatially white. The sampling interval $T_{s}$ for obtaining data snapshots is set to $0.1, L_{b}=100$, and $q=1.6$. The initial matrix $\hat{\mathbf{R}}_{x x}(0)$ is set to $10^{-6} \mathbf{I}$, where I denotes the identity matrix with size $21 \times 21$. The vector $\mathbf{c}$ for the LS-SCORE algorithm is fixed to $\mathbf{c}=[1,0, \cdots, 0]^{T}$. The time delay $\tau$ is set to 0 for simplicity. All the simulation results are obtained by averaging 50 independent runs with independent noise samples for each run.

Example 1: In this case, the CFE is $\Delta \alpha=-0.01$, i.e., $\hat{\alpha}=1.99$. Fig. 1 plots the simulation results in terms of the output SINR versus the number of snapshots. For comparison, the results of using the original LS-SCORE algorithm with and

$$
\Delta \alpha(i+1)\left\{(i+1) T_{s}\right\} \approx \frac{1}{2}+\frac{1}{2 i}-\underbrace{\left\{2^{4-2 q} \pi^{q-2} M^{-2 q} A_{s}^{4-2 q}\left\{\mathbf{a}_{d}^{H} \hat{\mathbf{R}}_{x x}^{-1}(i) \mathbf{a}_{d}\right\}\left\{\mathbf{c}^{H} \mathbf{a}_{d}^{*} \mathbf{a}_{d}^{T} \mathbf{c}\right\}\left(1+\frac{\pi^{2}}{4}\right)^{-q / 2} T_{s}^{2-q}\right\}}_{C_{2}} \frac{i+1}{i^{q-1}}
$$

$$
\begin{aligned}
\Delta \alpha(i+1)\left\{(i+1) T_{s}\right\} & \begin{cases}\frac{-1}{2}-\frac{1}{2 i}+\underbrace{\left\{2^{4-2 q} \pi^{q-2} M^{-2 q} A_{s}^{4-2 q}\left\{\mathbf{a}_{d}^{H} \hat{\mathbf{R}}_{x x}^{-1}(i) \mathbf{a}_{d}\right\}\left\{\mathbf{c}^{H} \mathbf{a}_{d}^{*} \mathbf{a}_{d}^{T} \mathbf{c}\right\} T_{s}^{2-q}\right\}}_{C_{1}} \frac{i+1}{i^{q-1}}, & \text { if } t_{0}=-\frac{T}{2} \\
\frac{-1}{2}-\frac{1}{2 i}+\underbrace{\left\{2^{4-2 q} \pi^{q-2} M^{-2 q} A_{s}^{4-2 q}\left\{\mathbf{a}_{d}^{H} \hat{\mathbf{R}}_{x x}^{-1}(i) \mathbf{a}_{d}\right\}\left\{\mathbf{c}^{H} \mathbf{a}_{d}^{*} \mathbf{a}_{d}^{T} \mathbf{c}\right\}\left(1+\frac{\pi^{2}}{4}\right)^{-q / 2} T_{s}^{2-q}\right\}}_{C_{2}} \frac{i+1}{i^{q-1}}, & \text { if } t_{0}=\text { a constant }\end{cases}
\end{aligned}
$$




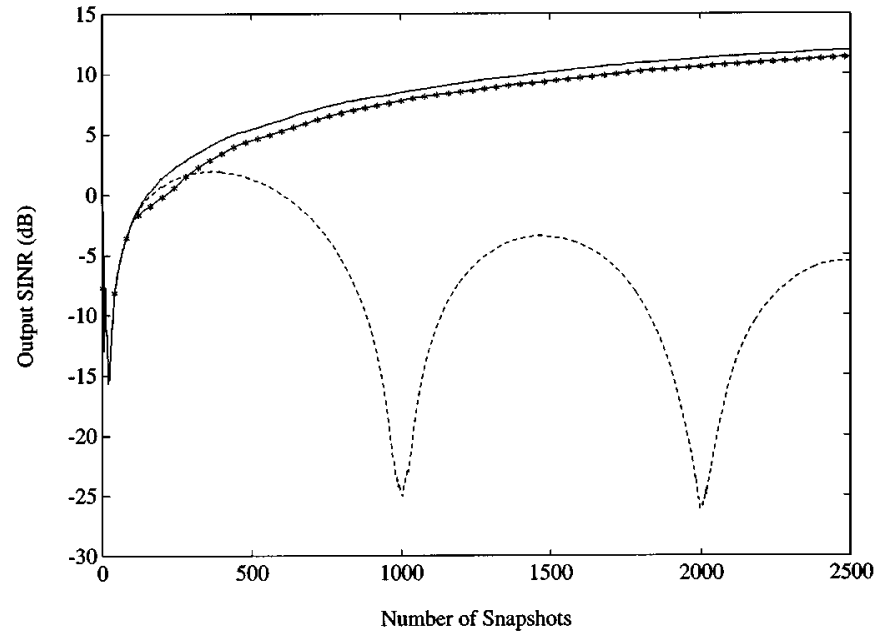

- The original LS-SCORE algorithm when $\hat{\alpha}=2.0$.
--- : The original LS-SCORE algorithm when $\hat{\alpha}=1.99$.
$-*-*$ : The proposed method based on LS-SCORE algorithm with $q=1.6$ when
$\hat{\alpha}=1.99$.

Fig. 1. Output SINR versus number of snapshots for Example 1. — : The original LS-SCORE algorithm when $\hat{\alpha}=2.0$. ---- : The original LS-SCORE algorithm when $\hat{\alpha}=1.99 .-*-*$ : The proposed method based on LS-SCORE algorithm with $q=1.6$ when $\hat{\alpha}=1.99$.

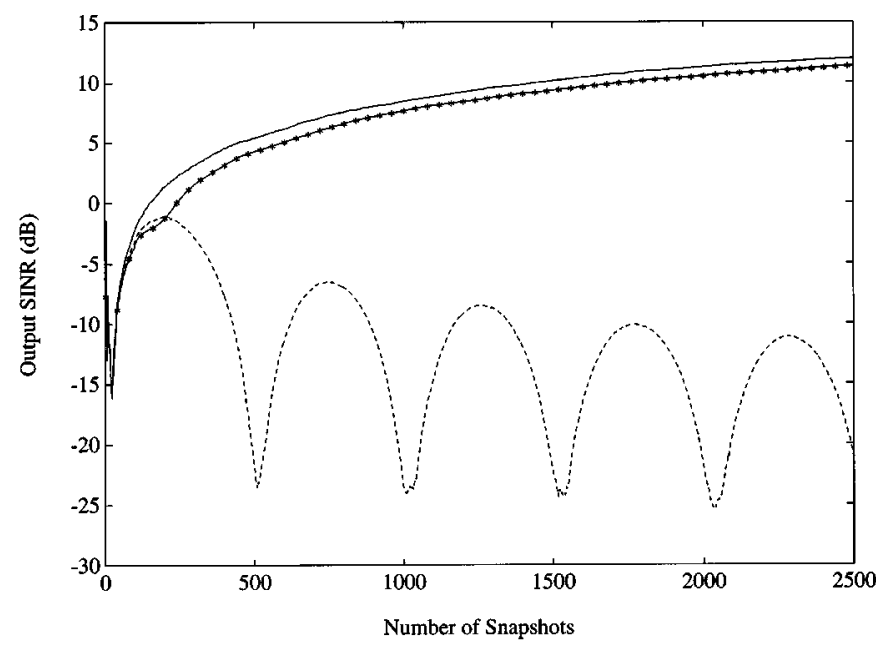

- The original LS-SCORE algorithm when $\hat{\alpha}=2.0$.

--- : The original LS-SCORE algorithm when $\hat{\alpha}=2.0197$.

- * -*: The proposed method based on LS-SCORE algorithm with $q=1.6$ when $\hat{\alpha}=2.0197$.

Fig. 2. Output SINR versus number of snapshots for Example 2.

The original LS-SCORE algorithm when $\hat{\alpha}=2.0$. ---- : The original LS-SCORE algorithm when $\hat{\alpha}=2.0197 .-*-*$ : The proposed method based on LS-SCORE algorithm with $q=1.6$ when $\hat{\alpha}=2.0197$.

without CFE are also shown. We observe from this figure that the proposed method can effectively cope with the performance degradation due to the CFE and provides the performance very close to that of using the original LS-SCORE algorithm without CFE.

Example 2: Here, we repeat Example 1 with a different CFE $\Delta \alpha=0.0197$, i.e., $\hat{\alpha}=2.0197$. Fig. 2 depicts the corresponding simulation results of utilizing the proposed method.
For comparison, the results of using the original LS-SCORE algorithm with and without CFE are also shown. Again, we observe from this figure that the proposed method works satisfactorily in the circumstances with and without CFE, respectively.

Finally, we can see from the above figures that there exist almost periodic nulls for the original LS-SCORE algorithm with CFE when the number of data snapshots equal to $k /(0.01 \times$ $0.1)=1000 k, k=1,2, \cdots$ for Example 1 and $k /(0.0197 \times$ $0.1) \approx 500 k, k=1,2, \cdots$ for Example 2, respectively, as expected. Moreover, our experience shows that larger $q$ should be chosen in the presence of strong interference.

\section{CONCLUSION}

This paper has presented an efficient method for blind adaptive beamforming using the LS-SCORE algorithm in the presence of cycle frequency error (CFE). The performance degradation of the original LS-SCORE algorithm due to CFE has been analyzed. Based on the theoretical result, an efficient method in conjunction with the LS-SCORE algorithm has been developed to cope with the performance deterioration of the original LS-SCORE algorithm due to CFE. According to the proposed method, an appropriate estimate of the cycle frequency of the desired signal is first found based on the use of steepest-descent algorithm. Using the estimate, we then construct new reference signal required by the LS-SCORE algorithm. The convergence property regarding the proposed method has been investigated. The validity of the theoretical works for performance analysis has been confirmed by simulation results. The effectiveness of the proposed method in dealing with adaptive beamforming for cyclostationary signals in the presence of CFE has also been demonstrated by simulation examples.

\section{REFERENCES}

[1] R. A. Monzingo and T. W. Miller, Introduction to Adaptive Array. New York: Wiley, 1980.

[2] W. A. Gardner, "Exploitation of spectral redundancy in cyclostationary signals," IEEE Signal Processing Mag., pp. 14-36, Apr. 1991.

[3] — "Spectral correlation of modulated signals: Part I-Analog modulation," IEEE Trans. Commun., vol. COMM-35, pp. 584-594, June 1987.

[4] W. A. Gardner, W. A. Brown, III, and C.-K. Chen, "Spectral correlation of modulated signals: Part II-Digital modulation," IEEE Trans. Commun., vol. COMM-35, pp. 595-601, June 1987.

[5] W. A. Gardner, Cyclostationarity in Communications and Signal Processing. New York: IEEE Press, 1994.

[6] B. G. Agee, S. V. Schell, and W. A. Gardner, "Spectral self-coherence restoral: A new approach to blind adaptive signal extraction using antenna arrays," Proc. IEEE, vol. 78, pp. 753-767, Apr. 1990.

[7] S. V. Schell, "Performance analysis of the cyclic MUSIC method of direction estimation for cyclostationary signals," IEEE Trans. Signal Processing, vol. 42, pp. 3043-3050, Nov. 1994.

[8] - "Asymptotic moments of estimated cyclic correlation matrices," IEEE Trans. Signal Processing, vol. 43, pp. 173-180, Jan. 1995.

[9] S. V. Schell and W. A. Gardner, "Progress on signal-selective direction finding," in Proc. 5th Acoust., Speech, Signal Process. Workshop Spectrum Estim. Modeling, Rochester, NY, Oct. 1990, pp. 144-148.

[10] T. E. Biedka and B. G. Agee, "Subinterval cycle MUSIC-robust DF with error in cycle frequency knowledge," in Proc. 25th Asilomar Conf. Signals, Syst., Comput., Pacific Grove, CA, Nov. 1991, pp. 262-266.

[11] S.-J. Yu and J.-H. Lee, "Adaptive array beamforming for cyclostationary signals," IEEE Trans. Antennas Propagat., vol. 44, pp. 943-953, July 1996. 


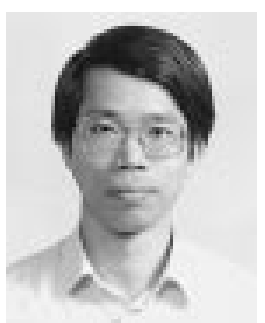

Ju-Hong Lee was born in I-Lan, Taiwan, R.O.C., on December 7, 1952. He received the B.S. degree from the National Cheng-Kung University, Tainan, Taiwan, in 1975, the M.S. degree from the National Taiwan University (NTU), Taipei, Taiwan, in 1977, and the Ph.D. degree from Rensselaer Polytechnic Institute (RPI), Troy, NY, in 1984, all in electrical engineering.

From September 1980 to July 1984, he was a Research Assistant and was involved in research on multidimensional recursive digital filtering with the Department of Electrical, Computer, and Systems Engineering at RPI. From August 1984 to July 1986, he was a Visiting Associate Professor and, in August 1986, became an Associate Professor in the Department of Electrical Engineering, NTU. Since August 1989, he has been a Professor at the same university. He was appointed Visiting Professor with the Department of Computer Science and Electrical Engineering, University of Maryland, Baltimore County, during a sabbatical leave in 1996. His current research interests include multidimensional digital signal processing, image processing, detection and estimation theory, analysis and processing of joint vibration signals for the diagnosis of cartilage pathology, and adaptive signal processing and its applications in communications.

Dr. Lee received Outstanding Research Awards from the National Science Council (NSC) in the academic years of 1988, 1989, and 1991-1994, and Distinguished Research Awards from the NSC in the academic years of 1998 and 1999.

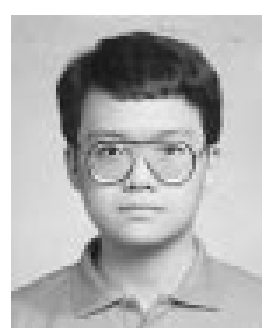

Yung-Ting Lee was born in Tainan, Taiwan, R.O.C., on March 31, 1971. He received the B.S. degree from the National Cheng-Kung University, Tainan, in 1993 and the M.S. and the Ph.D. degrees from the National Taiwan University, Taipei, in 1995 and 2000, respectively, all in electrical engineering.

His current research interests include adaptive signal processing, array signal processing, and the theory and application of cyclostationarity.

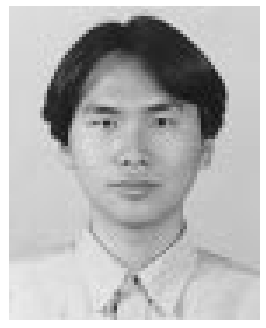

Wen-Hao Shih was born in Yunlin, Taiwan, R.O.C., on November 4, 1973. He received the B.S. and the M.S. degrees from the National Taiwan University, Taipei, Taiwan, in 1996 and 1998, respectively, both in electrical engineering.

His current research interests include adaptive signal processing and array signal processing and its applications in communications. 